\title{
Three-dimensional finite element analysis of composites with coated spherical inclusions
}

\author{
Yongli $\mathrm{Wu}$, Zhifa Dong \\ Institute of Mechanics, Chinese Academy of Sciences, Beijing 100080, China
}

Received 14 October 1994; in revised form 22 March 1995

\begin{abstract}
A three-dimensional finite element analysis has been used to determine the internal stresses in a three-phase composite. The stresses have been determined for a variety of interphase properties, the thicknesses of the interphase and the volume fractions of particles. Young's modulus has been calculated from a knowledge of these stresses and the applied deformation. The calculations show that stress distributions in the matrix and the mechanical properties are sensitive to the interphase property in the three-phase composites. The interfacial stresses in the three-dimensional analysis are in agreement with results obtained by an axisymmetric analysis. The predicted bulk modulus in three-dimensional analysis agrees well with the theoretical solution obtained by Qui and Weng, but it presents a great divergence from that in axisymmetric analyses. An investigation indicates that this divergence may be caused by the difference in the unit cell structure between two models. A comparison of the numerically predicted bulk and shear modulus for two-phase composites with the theoretical results indicates that the three-dimensional analysis gives quite satisfactory results.
\end{abstract}

Keywords: Finite element method; Three-phase composites; Interphase; Stress field

\section{Introduction}

This paper is concerned with the micromechanical analysis of three-phase particulate-reinforced composites, which consist of particles as one phase (inclusions), coatings as a second phase (interphase) in which thickness depends on the chemistry and processing conditions of the particles and a third phase, the matrix. The interphase, generally a low modulus elastomer, is added to solve the problem of the two-phase composites which, when containing a certain volume fraction of high modulus inclusions, exhibit an increase in overall modulus and tensile strength but a considerable reduction in toughness, and thus are expected to develop the mechanical property of the two-phase composite.

For the particulate-reinforced composites in the absence of interphase, many investigators have made considerable efforts to analyse the stress fields of composites and to determine the mechanical properties of this kind of material. Hashin [1] and Christensen and
Lo [2] used the composite sphere model to present this material and give its effective shear and bulk moduli by the self-consistent scheme. Weng [3] and Luo and Weng [4], on the basis of the "average stress in the matrix" concept of Mori and Tanaka [5], developed a useful modified Mori-Tanaka method to predict the overall moduli of the composites. Agarwal and Broutman [6] gave a three-dimensional finite element solution for the stress fields of constituent phases, stress concentrations at the interface and a prediction of the mechanical properties. However, there are only a few solutions available for composites in the presence of an interphase. The earlier Matonis and Small [7] solution, which was an extension of Goodier's work [8], applies only to the case of a single inclusion with coating layer in an infinite matrix. The theory ignored the interactions between particles. The recent Qiu and Weng [9] solution determines the effective elastic moduli of thickly coated particle and fibre-reinforced composites on the basis of the models of Hashin [1] and Hashin and Rosen [10]. Benveniste et al. [11] also give a 
solution for the stress fields in the fibre-reinforced composite with coated inclusions by the modified Mori-Tanaka method.

As a result of the presence of interphase, the matrix plastic deformation is restricted by the particles and interphases. The magnitude of this restraint is complex and is a function of interparticle spacings, volume fractions of particles, aspects of the interphase and the elastic properties of the matrix, particle and interphase. Thus a micromechanics analysis is needed to understand the influence of these factors in the behaviour of the materials. Here, we use the finite element method to determine the three-dimensional elastic stress distribution of the heterogeneous composite structures. The internal stress is used to predict elastic properties such as modulus and Poisson's ratio. The results are compared with the values predicted by an axisymmetric analysis.

\section{Material model and boundary conditions}

In a real composite, particles are randomly dispersed in the matrix. The mechanical behaviour of the composite is dependent on the physical properties, relative amounts and packing geometry of the constituent phases and on the condition of the interfaces between these phases. For convenience of studies, the constituents are assumed to be isotropic. Perfect bonding is assumed at the interfaces between particle and interphase and between interphase and matrix. Spherical inclusions are assumed to be packed in a cubic array, as shown in Fig. 1(a). Because of the assumed symmetry for the packing of the spherical particles, we need only to analyse one-eighth of the sphere embedded in a cube, as shown in Fig. 1(b). The unit cell model and one of its finite element grids used in the present work are shown in Fig. 2. The grid shown is for the case where the composite contains a filler volume fraction of $23.56 \%$ $\left(r_{1} / r=0.7663\right)$. The filler volume fraction, thickness of interphase and interphase volume fraction in the model are given by the following: volume fraction of particles,

$V_{\mathrm{i}}=\frac{\pi}{6}\left(\frac{r_{1}}{r}\right)^{3}$

thickness of interphase,

$h=r_{2}-r_{1}$

volume fraction of interphase,

$V_{\mathrm{f}}=\frac{\pi}{6}\left[\left(\frac{r_{2}}{r}\right)^{3}-\left(\frac{r_{1}}{r}\right)^{3}\right]$

Here $r$ is the cell length, $r_{1}$ the particle radius and $r_{2}$ the outer radius of interphases depicted in Fig. 1(b). For the sake of comparison, we also carry out an axisymmetric analysis of the composite. Fig. 3(a) shows the packing and structure of the axisymmetric cell, in which the cube becomes a solid circular bar. The particle is embedded in an axisymmetric solid. The corresponding finite element mesh is shown in Fig. 3(b).

The boundary conditions and solution procedures used follow those of Agarwal and Broutman [6]. The unit cell as shown in Fig. 1(b) is subjected to a uniaxial tension in the $z$ direction and no tractions in the $x$ and $y$ directions. By symmetry, the shear stresses $\tau_{x y}, \tau_{y z}, \tau_{z x}$ on all the faces of the cube must be zero, and thus the faces $\mathrm{ABFE}, \mathrm{EFGH}$ and $\mathrm{BCGF}$ of the cube remain parallel to their original position while the faces $A B C D$, $\mathrm{ADHE}$ and DCGH remain fixed. In addition, no external forces are acting on the faces ABFE and BCGF, so that

$\int_{A} \sigma_{x} \mathrm{~d} A=0 \quad$ on $x=r$
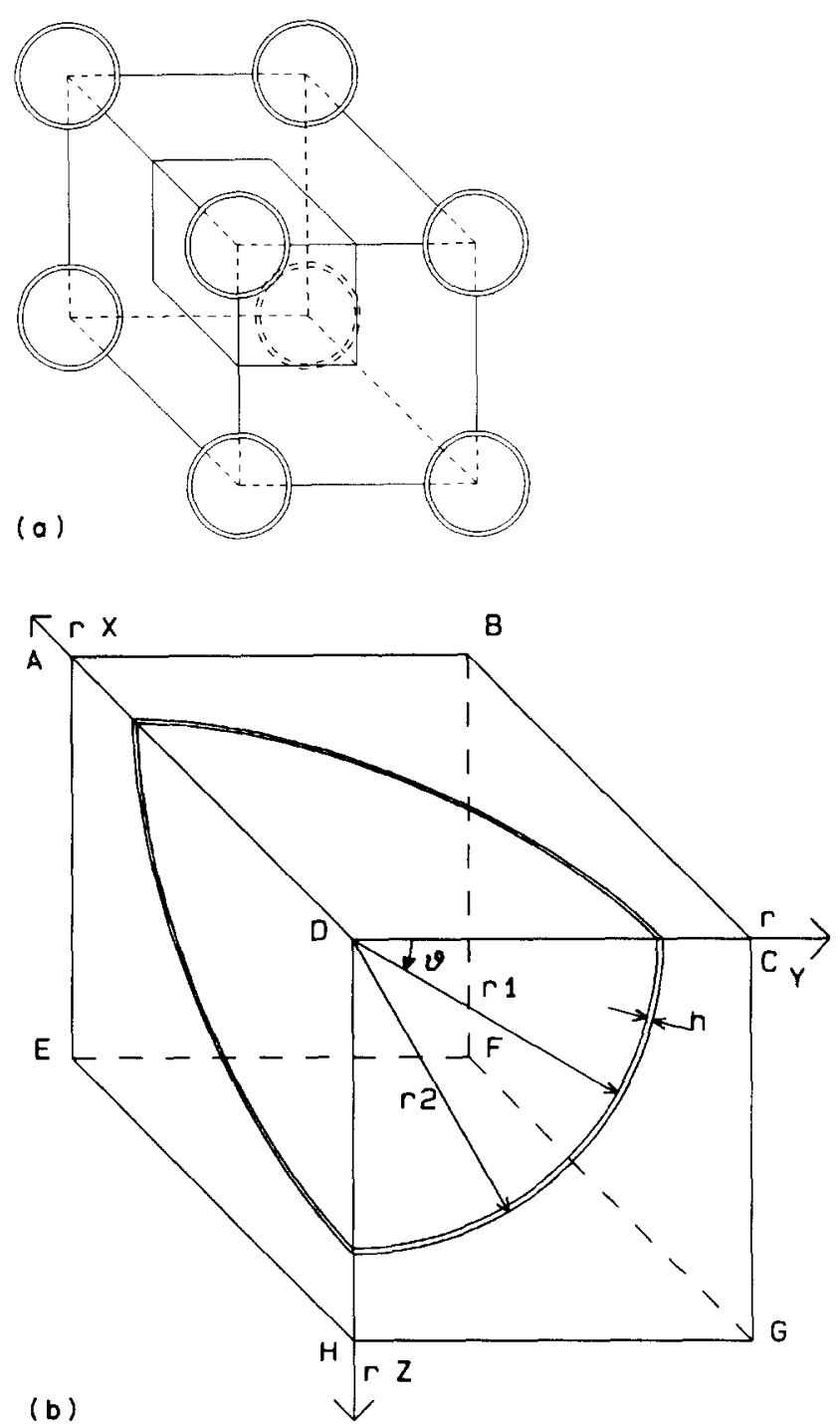

Fig. 1. (a) Packing of spherical inclusions in a composite. (b) A three-dimensional unit cell analysed. 


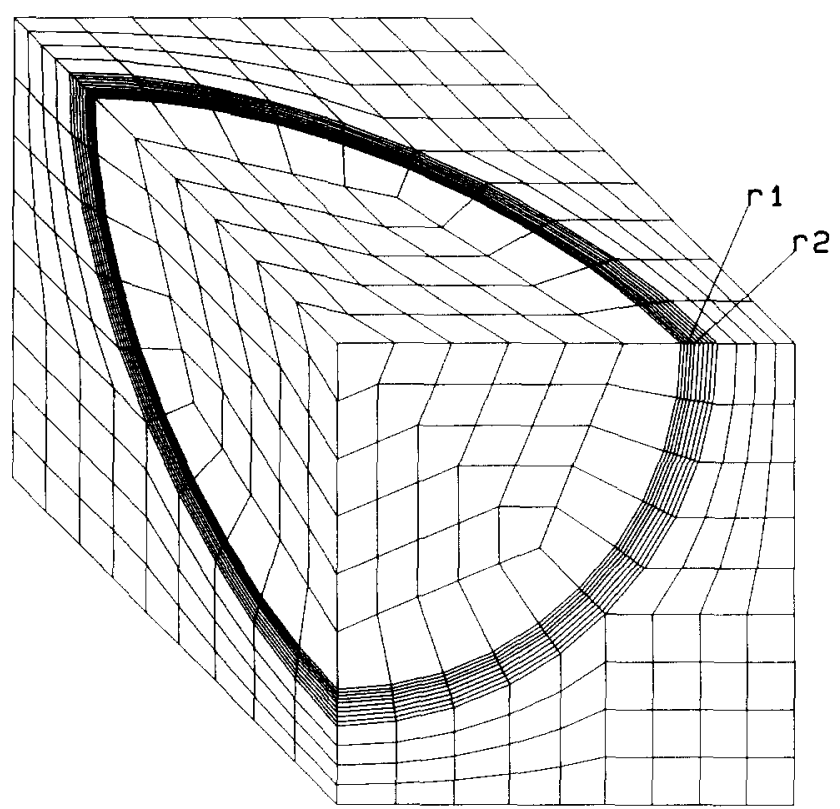

Fig. 2. Three-dimensional mesh for $r_{1} / r=0.7663$.

$\int_{A} \sigma_{y} \mathrm{~d} A=0 \quad$ on $y=r$

These boundary conditions are satisfied by the superposition of three separate stress analysis problems. In the first problem, the model is given zero displacement on all boundaries except for the face ABFE which is subjected to a unit (positive) displacement in the $x$ direction. In the second, the cell is subjected to zero displacements on all faces except for the face BCGF which is given a unit displacement in the $y$ direction. In the third, stresses and displacements are calculated when the face EFGH is subjected to a unit normal displacement in the $z$ direction while other faces are given zero displacements. Then the stresses and displacements predicted in the above three cases are superimposed by

$\sigma=k_{1} \sigma_{1}+k_{2} \sigma_{2}+\sigma_{3}$

$U=k_{1} U_{1}+k_{2} U_{2}+U_{3}$

where $k_{1}$ and $k_{2}$ are determined such that boundary conditions (4) and (5) are satisfied:

$$
\begin{aligned}
& \int_{\mathrm{ABFE}}\left(k_{1} \sigma_{x 1}+k_{2} \sigma_{x 2}+\sigma_{x 3}\right) \mathrm{d} A \\
& =A\left(k_{1} \bar{\sigma}_{x 1}+k_{2} \bar{\sigma}_{x 2}+\bar{\sigma}_{x 3}\right)=0 \\
& \int_{\mathrm{BCGF}}\left(k_{1} \sigma_{y 1}+k_{2} \sigma_{y 2}+\sigma_{y 3}\right) \mathrm{d} A \\
& =A\left(k_{1} \bar{\sigma}_{y 1}+k_{2} \bar{\sigma}_{y 2}+\bar{\sigma}_{y 3}\right)=0
\end{aligned}
$$

so

$$
k_{1}=-\frac{\bar{\sigma}_{y 2} \bar{\sigma}_{x 3}-\bar{\sigma}_{x 2} \bar{\sigma}_{y 3}}{\bar{\sigma}_{x 1} \bar{\sigma}_{y 2}-\bar{\sigma}_{y 1} \bar{\sigma}_{x 2}}, \quad k_{2}=-\frac{\bar{\sigma}_{x 1} \bar{\sigma}_{y 3}-\bar{\sigma}_{y 1} \bar{\sigma}_{x 3}}{\bar{\sigma}_{x 1} \bar{\sigma}_{y 2}-\bar{\sigma}_{y 1} \bar{\sigma}_{x 2}}
$$

where $A$ is the area of the plane ABFE or BCGF. Eqs. (1), (5), (8), (9) and (10) allow for the evaluation of Poisson's ratio as

$v_{z x}=\left|k_{1}\right|, \quad v_{z y}=\left|k_{2}\right|$

The modulus of elasticity is defined as

$E=\bar{\sigma}_{z} / \bar{\varepsilon}_{z}$
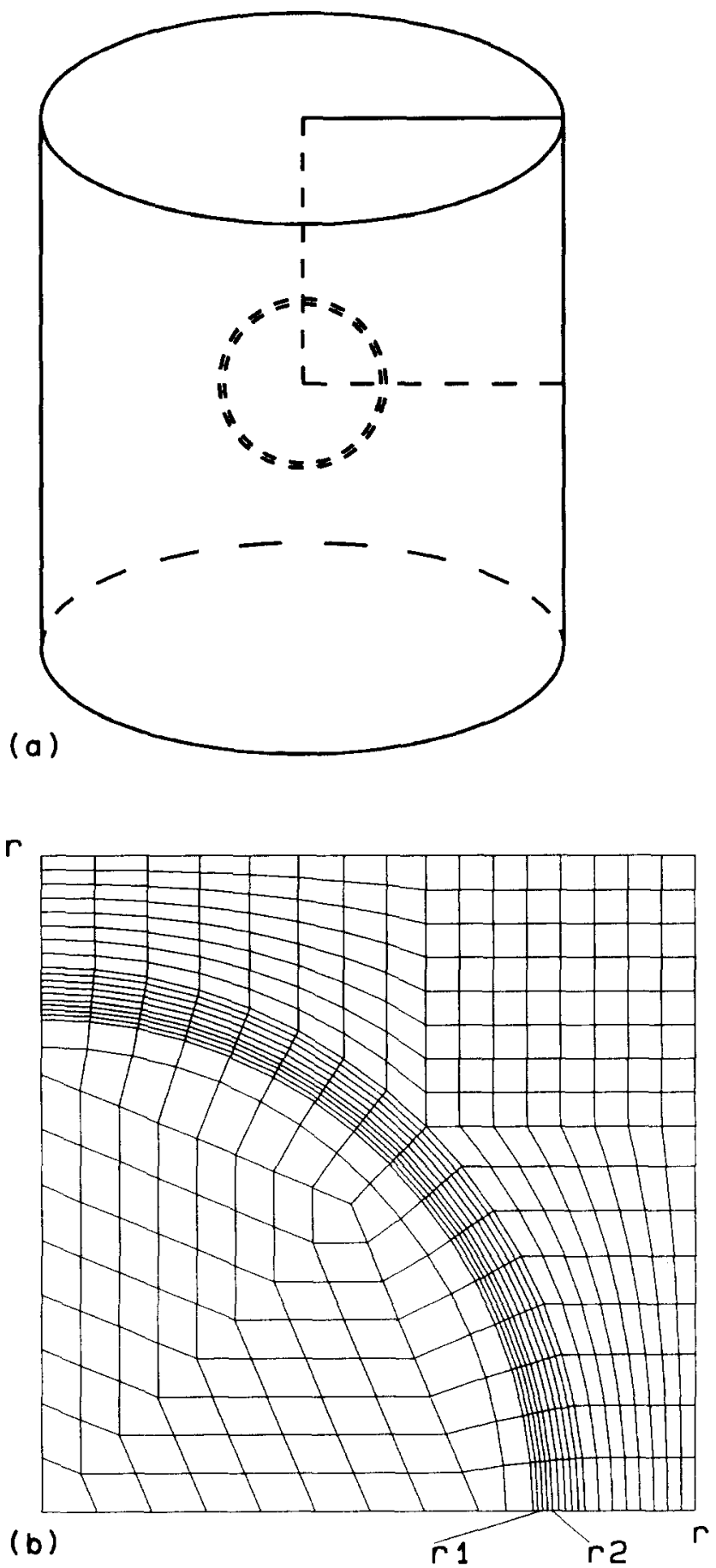

Fig. 3. (a) Packing of the axisymmetric cell in composites. (b) Axisymmetric mesh for $r_{1} / r=0.7663$. 
where $\bar{\varepsilon}_{z}$ is the strain in the $z$ direction. The stress $\bar{\sigma}_{z}$ is calculated as

$\bar{\sigma}_{z}=\frac{\int_{A} \sigma_{z} \mathrm{~d} A}{A}=\frac{1}{A} \sum_{i=1}^{n} \sigma_{z} A_{i}$

where $\sigma_{z}$ is the stress in an element and $A_{i}$ is the area of the element on the face EFGH and $n$ is the total number of such elements. In this paper Eqs. (4)-(13) are taken from Ref. [6].

In order to appreciate better the magnitude of the triaxial stresses acting locally in the composite which can induce deformation, the von Mises equivalent stress $\sigma_{\mathrm{e}}$ is calculated as

$\sigma_{\mathrm{e}}=\left\{\frac{1}{2}\left[\left(\sigma_{1}-\sigma_{2}\right)^{2}+\left(\sigma_{2}-\sigma_{3}\right)^{2}+\left(\sigma_{3}-\sigma_{1}\right)^{2}\right]\right\}^{1 / 2}$

where $\sigma_{1}, \sigma_{2}$ and $\sigma_{3}$ are the principal stresses. In addition, the hydrostatic component of the stress is also calculated at the interfaces:

$\sigma_{\mathrm{m}}=\left(\sigma_{1}+\sigma_{2}+\sigma_{3}\right) / 3$

\section{Internal stresses}

The internal triaxial stresses were calculated throughout the volume of representative three-phase composite for a variety of interphase properties, interphase thicknesses and volume fractions of particles. The following component property ratios were assumed: Young's modulus, $E_{\mathrm{i}}$ (inclusion): $E_{\mathrm{f}}$ (interphase): $E_{\mathrm{n}}$ (matrix) $=35$ : $0.05: 1$; Poisson's ratio, $v_{\mathrm{i}}: v_{\mathrm{f}}: v_{\mathrm{m}}=0.23: 0.48: 0.40$. These property ratios are equivalent to the experimental values reported by Lu et al. [12].

\subsection{Stress distributions in the matrix}

The stresses $\sigma_{1}, \sigma_{\mathrm{e}}$ represented here were normalized by $E_{\mathrm{m}} \varepsilon_{z}$ for the convenience of comparisons. Because of the geometrical symmetry of the unit cell, only the stresses on the $x=0$ plane (CDGH) are chosen in this analysis. Fig. 4 shows the maximum principal stress contours in the matrix predicted by the three-dimensional analysis (Fig. 4(a)) and the axisymmetric analysis (Fig. 4(b)) for $V_{\mathrm{i}}=30 \%$ and $h=0.015 r$. Fig. 5 shows the corresponding von Mises stress contours in the matrix for two analyses. The figures show that the two analyses give almost identical stress distribution trends in the polar region, even though the values of the stress $\sigma_{1}$ in the three-dimensional analysis are slightly higher than those in the axisymmetric analysis, 5.4 vs. 5.1. In the matrix near the equatorial area as shown in Fig. 4, it is clear that a local stress island, that is, a local stress unevenness, exists in the stress $\sigma_{1}$ contour predicted by the axisymmetric analysis, but the solutions of the three-dimensional analysis are smoothly changed along
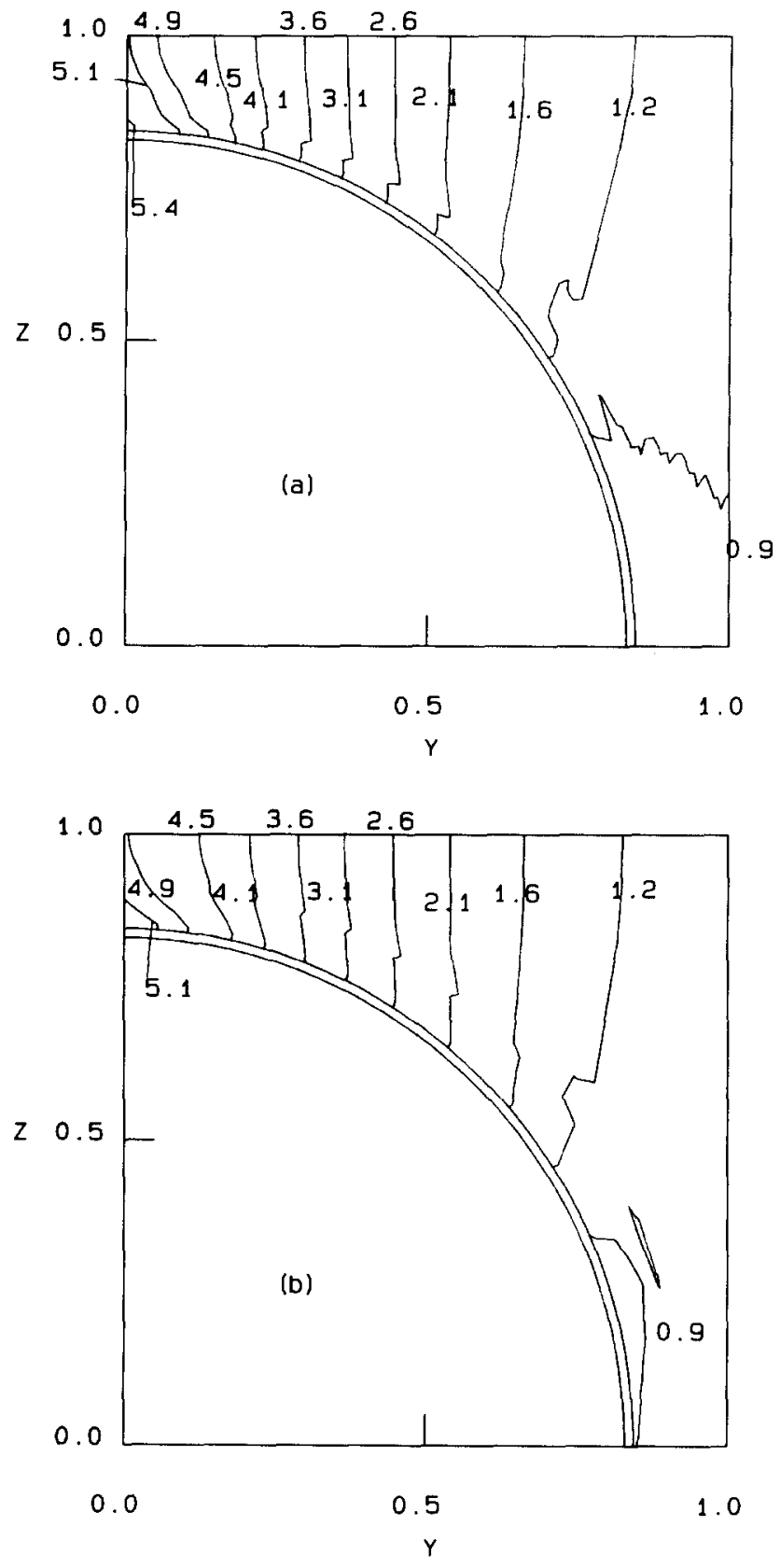

Fig. 4. Comparison of the maximum principal stress $\sigma_{1}$ contours in the matrix on the $x=0$ plane predicted by (a) three-dimensional and (b) axisymmetric analyses for $V_{\mathrm{i}}=30 \%, h=0.015 r . E_{\mathrm{i}}: E_{\mathrm{f}}: E_{\mathrm{m}}=$ 35.0:0.05:1.0, $v_{\mathrm{i}}: v_{\mathrm{f}}: v_{\mathrm{m}}=0.23: 0.48: 0.4 . \sigma_{1}$ was normalized by $E_{\mathrm{m}} \varepsilon_{z_{2}}$.

the interface. Comparing Fig. 5(a) with Fig. 5(b), one can also see that the stress values of the three-dimensional analysis in the matrix near the side surface of the axisymmetric bar (right upper area in the figures) are slightly larger than those of the axisymmetric analysis, 0.9 vs. 0.7 . The facts that an axisymmetric cell is only part of the three-dimensional cell, and thus a part of the matrix effects is neglected (for example, when $r_{2} /$ $r=0.7813,9 \%$ of the matrix is ignored), and because of the structure of the axisymmetric cell a free force boundary condition is acting on the side surface of the 
axisymmetric solid, make it reasonable to conclude that these stress differences in the matrix may be attributed to the difference in the unit cell structures in which the axisymmetric cells are not an actual repetitive unit but are related in their dimensions to the interparticle spacings $2\left(r-r_{1}\right)$. However, stresses at the interface between interphase and matrix are almost the same in the two analyses as shown in Fig. 6.

\subsection{Interface stresses}

In this section and Section 4, for considering the
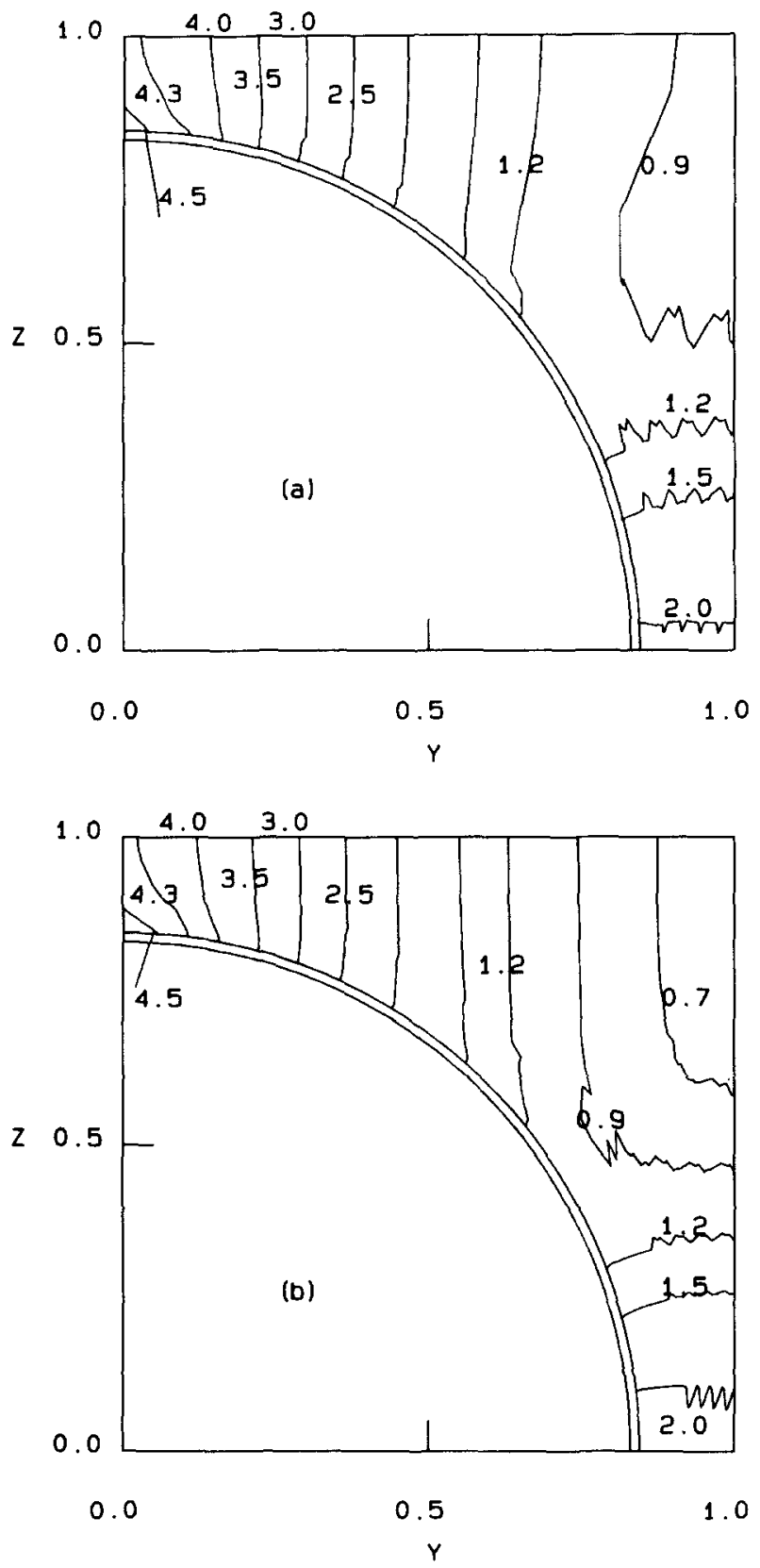

Fig. 5. Comparison of von Mises stress $\sigma_{\mathrm{e}}$ contours in the matrix on the $x=0$ plane predicted by (a) three-dimensional and (b) axisymmetric analyses for $V_{\mathrm{i}}=30 \%, h=0.015 \mathrm{r} . \quad E_{\mathrm{i}}: E_{\mathrm{f}}: E_{\mathrm{m}}=35.0: 0.05: 1.0$, $v_{\mathrm{i}}: v_{\mathrm{f}}: v_{\mathrm{m}}=0.23: 0.48: 0.4$. $\sigma_{\mathrm{e}}$ was normalized by $E_{\mathrm{m}} \varepsilon_{z}$.

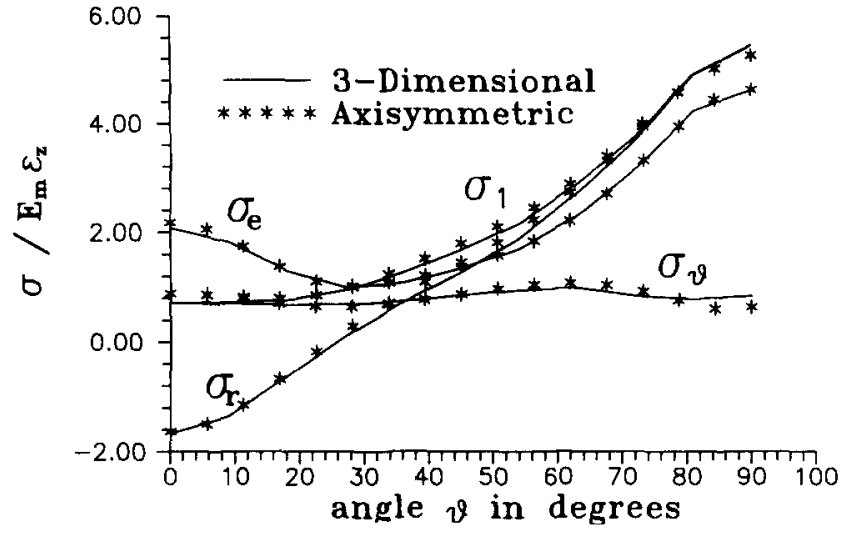

Fig. 6. Comparisons of stresses $\sigma_{1}, \sigma_{e}, \sigma_{r}, \sigma_{i,}$ in the matrix along the interface between interphase and matrix for the two analyses in Figs. 3 and 4.9 varies from $0^{\circ}$ to $90^{\circ}$ as shown in Fig. $1(\mathrm{~b})$.

effect of the interphase property on the stress distributions and mechanical properties of the composite, several different values of Young's modulus and Poisson's ratio of the interphase were assumed. Because of the geometrical symmetry of the unit cell, we only consider the stress on the $x=0$ plane $(\mathrm{CDGH})$. The stresses are represented as the ratio $\sigma_{i j} / \bar{\sigma}_{z}$, except for the case indicated, where $\bar{\sigma}_{z}$ is the average stress applied to the composite. At interfaces, the radial and tangential stresses are defined as

$\sigma_{r}=\frac{1}{2}\left(\sigma_{z}+\sigma_{y}\right)+\frac{1}{2}\left(\sigma_{y}-\sigma_{z}\right) \cos (2 \vartheta)+\tau_{y z} \sin (2 \vartheta)$

$\sigma_{\vartheta}=\frac{1}{2}\left(\sigma_{z}+\sigma_{y}\right)-\frac{1}{2}\left(\sigma_{y}-\sigma_{z}\right) \cos (2 \vartheta)-\tau_{y z} \sin (2 \vartheta)$

In a composite with a lower modulus interphase, the stresses around the interface between the interphase and the inclusion are shown in Fig. 7 for $V_{i}=23.56 \%$, $h=0.015 r$, where the hydrostatic pressures are the characteristic of the stress concentrations, and reach

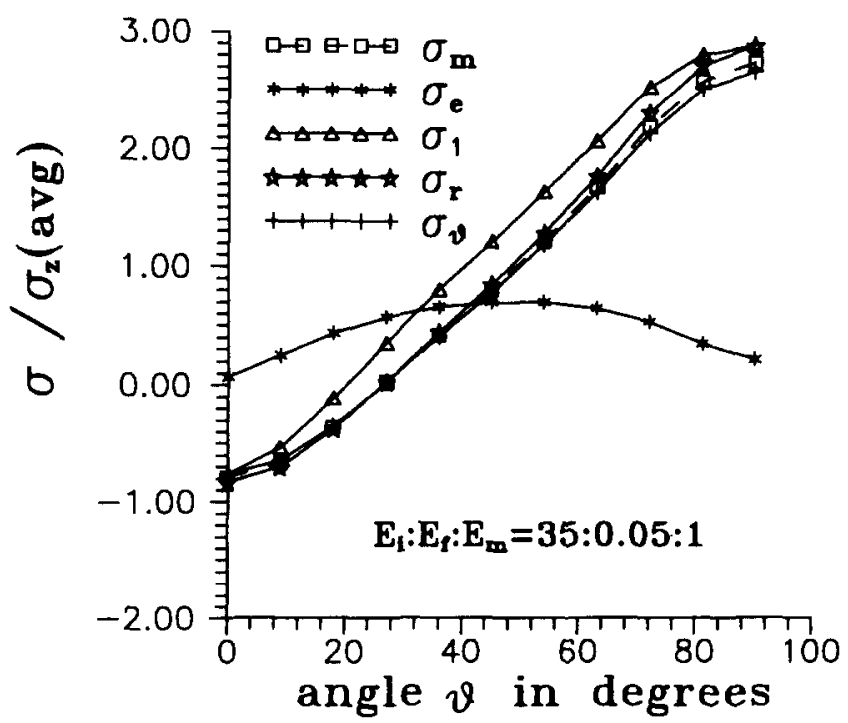

Fig. 7. Comparisons of stresses $\sigma_{1}, \sigma_{\mathrm{e}}, \sigma_{r}, \sigma_{9}$ in the interphase along the interface between particle and interphase for $r_{1} / r=0.7663$, $h=0.015 \mathrm{r}$. $\theta$ varies from $0^{\circ}$ to $90^{\circ}$ as shown in Fig. 1(b). 

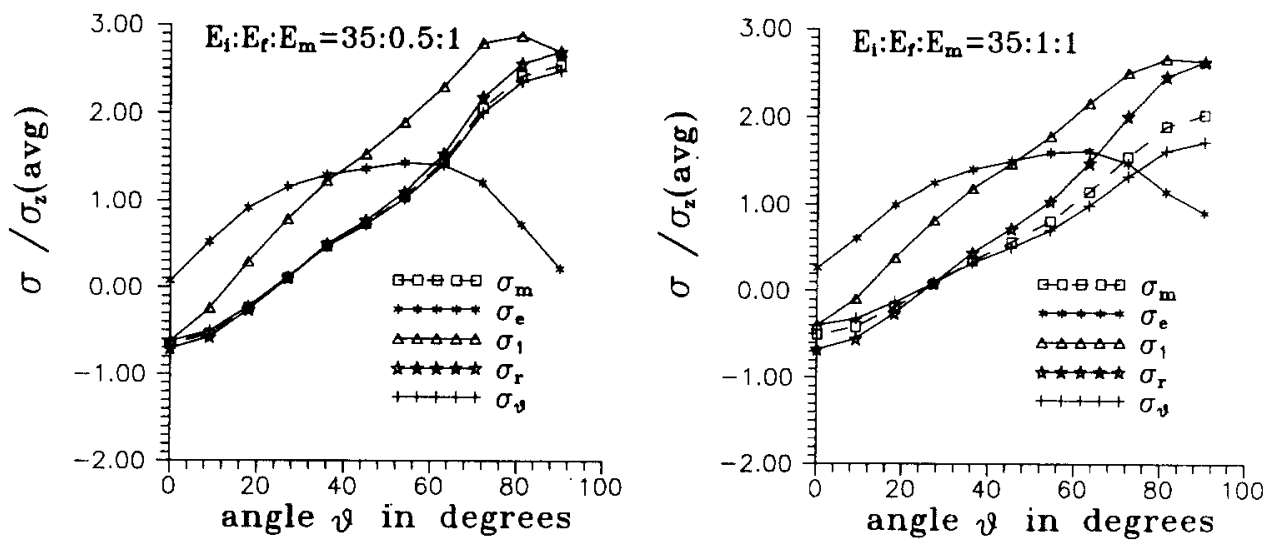

Fig. 8. Comparisons of stresses $\sigma_{1}, \sigma_{\mathfrak{e}}, \sigma_{r}, \sigma_{i\rangle}$ in the interphase along the interface between particle and interphase with different interphase properties for $r_{1}: r=0.7663, h=0.015 r$. 7 varies from $0^{\circ}$ to $90^{\circ}$ as shown in Fig. 1 (b)

maxima at the pole and minima at the equator. The calculations show that the stress distributions around the inclusions are weakly related to the interphase aspect ratios but very sensitive to the interphase property. Fig. 8 shows the stress distributions around the inclusion for different interphase properties. With the increase in elastic modulus of interphase, the stress contributions around the interfaces in three-phase com- posites are very similar to those in two-phase composites. It can be concluded that when the mechanical property of the interphase approaches that of the matrix, three-phase composites become similar to twophase composites.

Fig. 9 shows the matrix stress distributions around the interface between the interphase and matrix for different interphase properties. Great changes occur in the
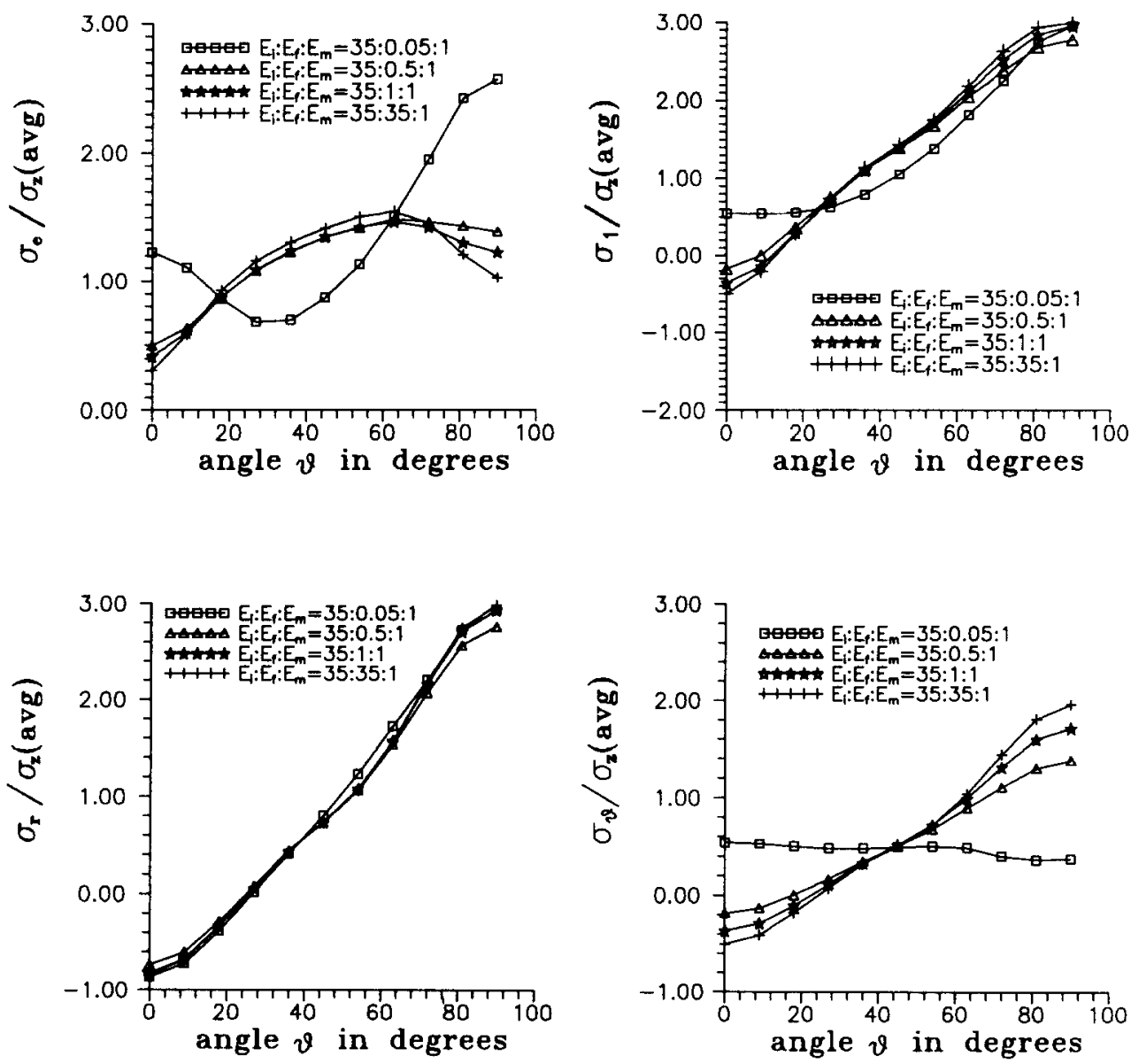

Fig. 9. Comparisons of stresses $\sigma_{1}, \sigma_{\mathrm{e}}, \sigma_{r}, \sigma_{i,}$ in the matrix along the interface between interphase and matrix with different interphase properties for $r_{1} / r=0.7663, h=0.015 r$. 9 varies from $0^{\circ}$ to $90^{\circ}$ as shown in Fig. $1(b)$. 

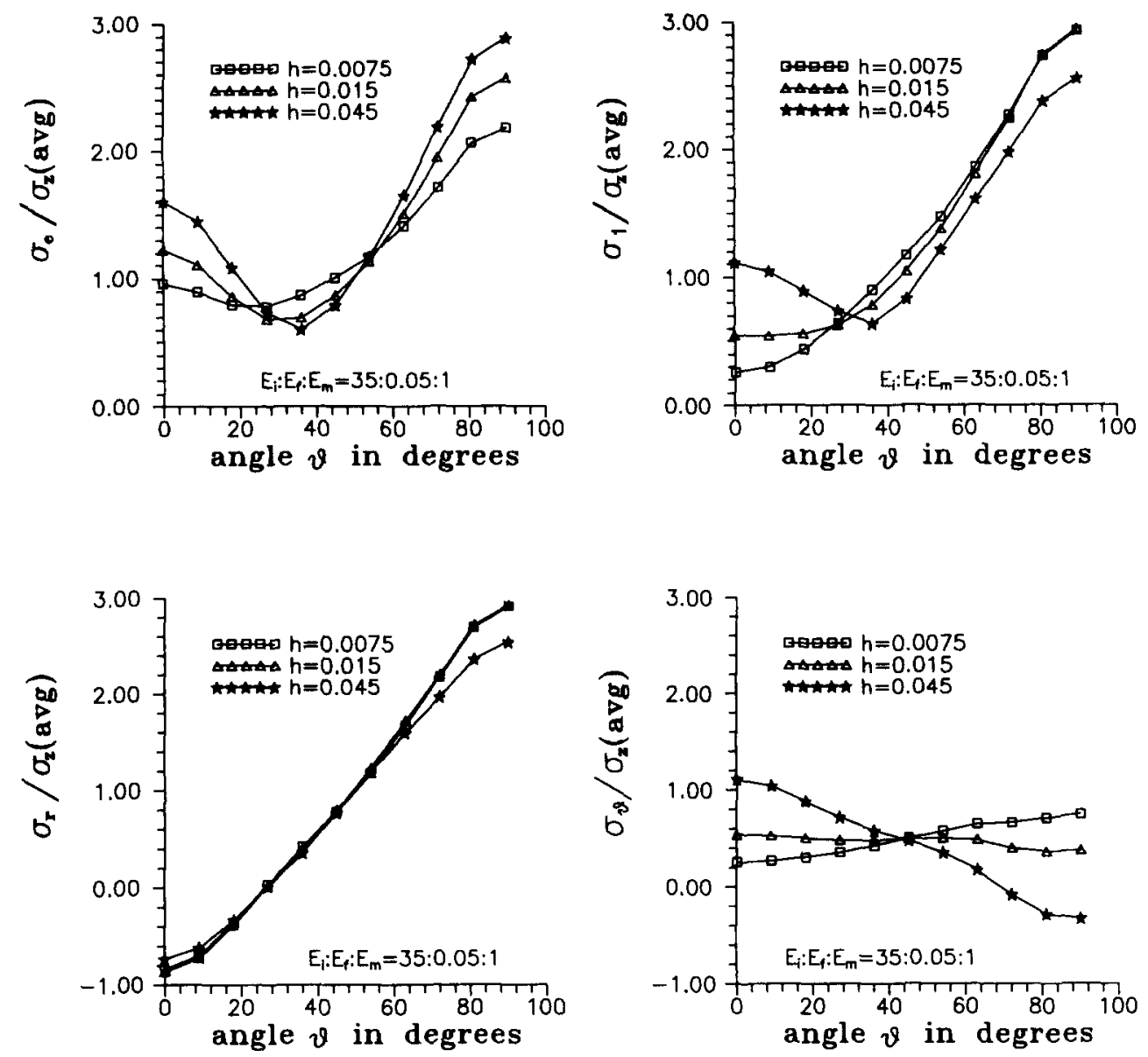

Fig. 10. Comparisons of stresses $\sigma_{1}, \sigma_{\mathrm{e}}, \sigma_{r}, \sigma_{9}$ in the matrix along the interface between interphase and matrix with various thicknesses of interphase for $r_{1} / r=0.7663,9$ varies from $0^{\circ}$ to $90^{\circ}$ as shown in Fig. 1(b).

von Mises stress and tangential stress along the interface. With the increase in the interphase modulus, the saddle-backed $\sigma_{\mathrm{e}}$ distributions for the low modulus interphase overturn the parabolic distributions for the high modulus interphase. The stress $\sigma_{\mathrm{e}}$ decreases both at the pole and at the equator. The tangential stress increases at the pole and decreases at the equator as the interphase modulus increases.

The stress concentrations along the interface between the interphase and matrix are also modified with the changes in the aspects of the interphase and inclusion. Fig. 10 shows the stress distributions for various thickness of the interphase. With the increase in the thickness, $\sigma_{\mathrm{e}}$ increases, but $\sigma_{1}, \sigma_{r}$ and $\sigma_{\vartheta}$ all decrease in the polar region, and, in the equatorial area, all of these stresses increase rapidly except $\sigma_{r}$ which has a minor increase. Fig. 11 shows the stress distributions around the interface between the interphase and matrix for various volume fractions of the inclusions. A great increase of the stresses occurs in the polar region when the filler content is up to $45 \%$, that is $r_{1} / r=0.9508$. This high polar stress concentration at the interface may be attributed to the strong interaction between adjacent particles for a high volume fraction of inclusions.

\section{Elastic constants}

Because the exact solution for the effective shear modulus is not available for the coated-particle-reinforced composite, although there is considerable information available on its bounds, we can only compare our results with Qiu and Weng's solution [9] of the effective bulk modulus for the material. To obtain the correct theoretical solution, it is necessary to let $c_{1}$ and $c_{2}$, the phase volume fractions defined in Ref. [9], be equal to $V_{\mathrm{i}}$ and $V_{\mathrm{f}}$ defined in Eqs. (1) and (3). Tables 1 , 2 and 3 show the predicted moduli of elasticity as a function of the interphase property, the thickness of the interphase and the volume fraction of inclusions respectively. For the given inclusion and matrix material, the elastic modulus of composites increases as the interphase property and the filler content increase but decreases as the thickness of the interphase increases. The calculations show that the bulk moduli predicted by three-dimensional analysis give a satisfactory agreement with the theoretical results.

Fig. 12 shows the bulk moduli obtained by threedimensional analysis, axisymmetric analysis and Qui and Weng's solution as a function of filler volume 

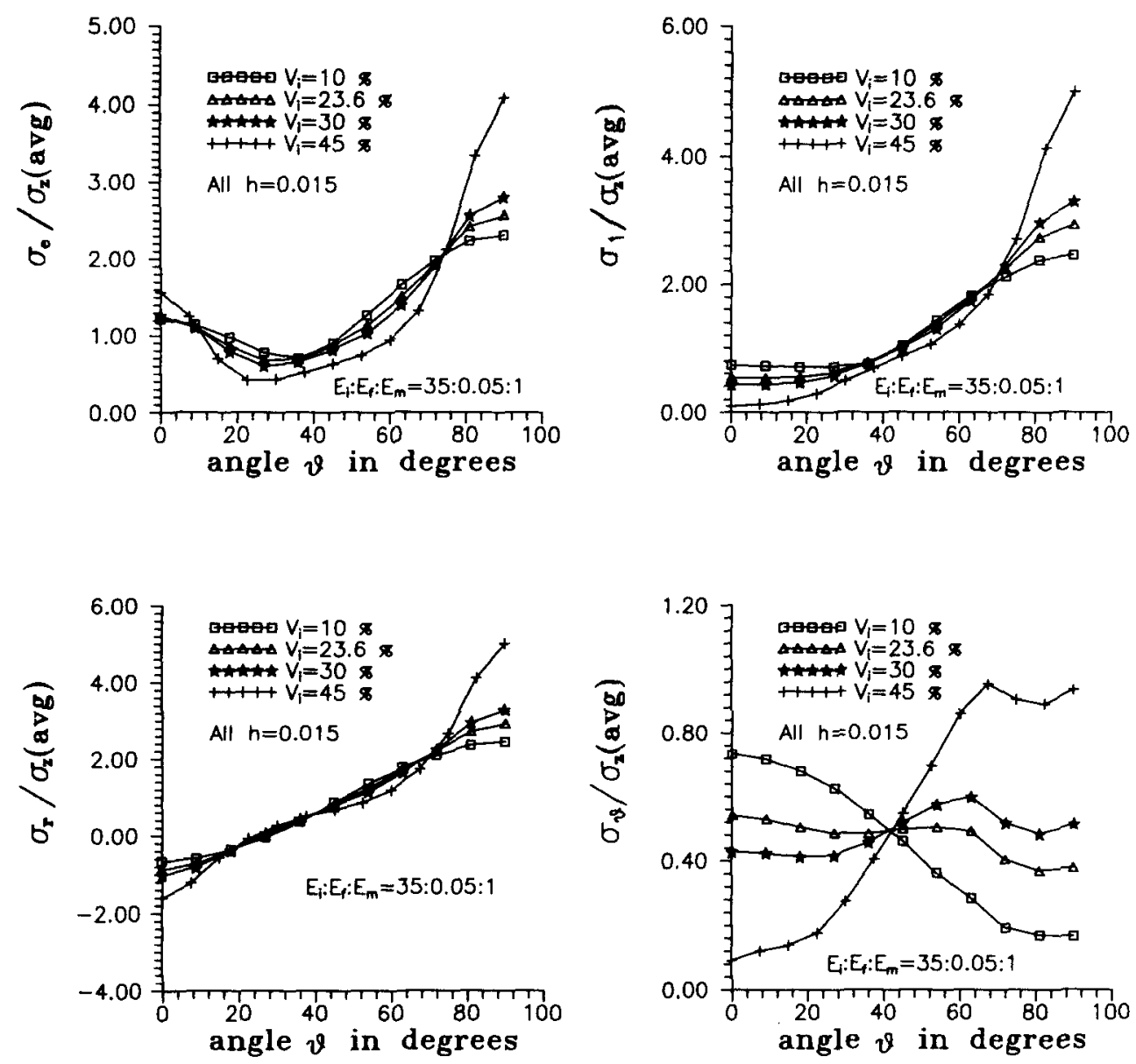

Fig. 11. Comparisons of stresses $\sigma_{1}, \sigma_{\mathrm{e}}, \sigma_{r}, \sigma_{\gamma}$ in the matrix along the interface between interphase and matrix with various volume fractions of particles. 9 varies from $0^{\circ}$ to $90^{\circ}$ as shown in Fig. 1(b).

Table 1

Predicted modulus of elasticity and bulk modulus of a three-phase composite as a function of interphase property for $h=0.015 r$, $V_{\mathrm{i}}=23.56 \%, V_{\mathrm{f}}=1.411 \%$, and $E_{\mathrm{i}}: E_{\mathrm{m}}=35.0: 1.0, v_{\mathrm{i}}: v_{\mathrm{m}}=0.23: 0.4$

\begin{tabular}{lllll}
\hline$E_{\mathrm{f}} / E_{\mathrm{m}}$ & $v_{\mathrm{f}}$ & $\begin{array}{l}\text { Present results } \\
\text { Eląstic modulus } \\
\text { ratio } \\
E / E_{\mathrm{m}}\end{array}$ & $\begin{array}{l}\text { Bulk } \\
\text { modulus } \\
k / E_{\mathrm{m}}\end{array}$ & $\begin{array}{l}\text { Qiu and Weng's } \\
\text { bulk modulus } \\
k / E_{\mathrm{m}}[9]\end{array}$ \\
\hline 0.05 & 0.48 & 1.4321 & 2.0737 & 2.0877 \\
0.5 & 0.48 & 1.8654 & 2.2545 & 2.2715 \\
1.0 & 0.40 & 1.9306 & 2.2237 & 2.2458 \\
35.0 & 0.23 & 2.0265 & 2.2683 & 2.2905 \\
\hline
\end{tabular}

fraction. In the axisymmetric analysis, we take the filler volume fraction as $V_{\mathrm{i}}=\frac{2}{3}\left(r_{1} / r\right)^{3}$. It should be noted that the definition of $V_{\mathrm{i}}$ in an axisymmetric analysis is related to the packing structure of the axisymmetric cells and a different arrangement gives a different definition [6]; however, such defined filler volume fraction is always larger than that defined in Eq. (1). From Fig. 12 , it is clear that the three-dimensional analysis agrees very well with the theoretical solution, but a great
Table 2

Predicted modulus of elasticity and bulk modulus of a three-phase composite as a function of interphase thickness for $V_{\mathrm{i}}=23.56 \%$, $E_{\mathrm{i}}: E_{\mathrm{f}}: E_{\mathrm{m}}=35.0: 0.05: 1.0, v_{\mathrm{i}}: v_{\mathrm{f}}: v_{\mathrm{m}}=0.23: 0.48: 0.40$

\begin{tabular}{|c|c|c|c|c|}
\hline \multirow[t]{2}{*}{$h / r$} & \multirow[t]{2}{*}{$V_{\mathrm{f}}$} & \multicolumn{2}{|l|}{ Present results } & \multirow{2}{*}{$\begin{array}{l}\text { Qiu and Weng's } \\
\text { bulk modulus } \\
k / E_{\mathrm{m}}[9]\end{array}$} \\
\hline & & $\begin{array}{l}\text { Elastic modulus } \\
\text { ratio } \\
E / E_{\mathrm{m}}\end{array}$ & $\begin{array}{l}\text { Bulk } \\
\text { modulus } \\
k / E_{\mathrm{m}}\end{array}$ & \\
\hline 0.075 & $0.699 \%$ & 1.5668 & 2.1457 & 2.1625 \\
\hline 0.015 & $1.411 \%$ & 1.4321 & 2.0730 & 2.0877 \\
\hline 0.045 & $4.399 \%$ & 1.1962 & 1.8375 & 1.8478 \\
\hline
\end{tabular}

difference between the three-dimensional analysis and the axisymmetric analysis exists for larger volume fractions. To understand better this difference between two analyses, the moduli of elasticity predicted by the threedimensional and axisymmetric analyses have both been plotted in Fig. 13 as a function of the ratio $r_{1} / r$ as suggested by Agarwal and Broutman [6]. It is seen that the results of the two analyses are quite close at lower volume fractions; however, at higher $r_{1} / r$ ratios, the moduli ratios predicted by the axisymmetric analysis 
Table 3

Predicted modulus of elasticity and bulk modulus of a three-phase composite as a function of volume fraction of inclusions for $h=0.015 r, E_{\mathrm{i}}: E_{\mathrm{f}}: E_{\mathrm{m}}=35.0: 0.05: 1.0, v_{\mathrm{i}}: v_{\mathrm{f}}: v_{\mathrm{m}}=0.23: 0.48: 0.40$

\begin{tabular}{lllll}
\hline$V_{\mathrm{i}}$ & $r_{1} / r$ & $\begin{array}{l}\text { Present results } \\
\text { Elastic modulus } \\
\text { ratio } \\
E / E_{\mathrm{m}}\end{array}$ & $\begin{array}{l}\text { Bulk } \\
\text { modulus } \\
k / E_{\mathrm{m}}\end{array}$ & $\begin{array}{l}\text { Qiu and Weng's } \\
\text { bulk modulus } \\
k / E_{\mathrm{m}}[9]\end{array}$ \\
\hline $10 \%$ & 0.5759 & 1.1256 & 1.8091 & 1.8114 \\
$23.56 \%$ & 0.7663 & 1.4321 & 2.0730 & 2.0877 \\
$30 \%$ & 0.8306 & 1.6546 & 2.2292 & 2.2490 \\
$45 \%$ & 0.9508 & 2.5571 & 2.7215 & 2.7269 \\
\hline
\end{tabular}

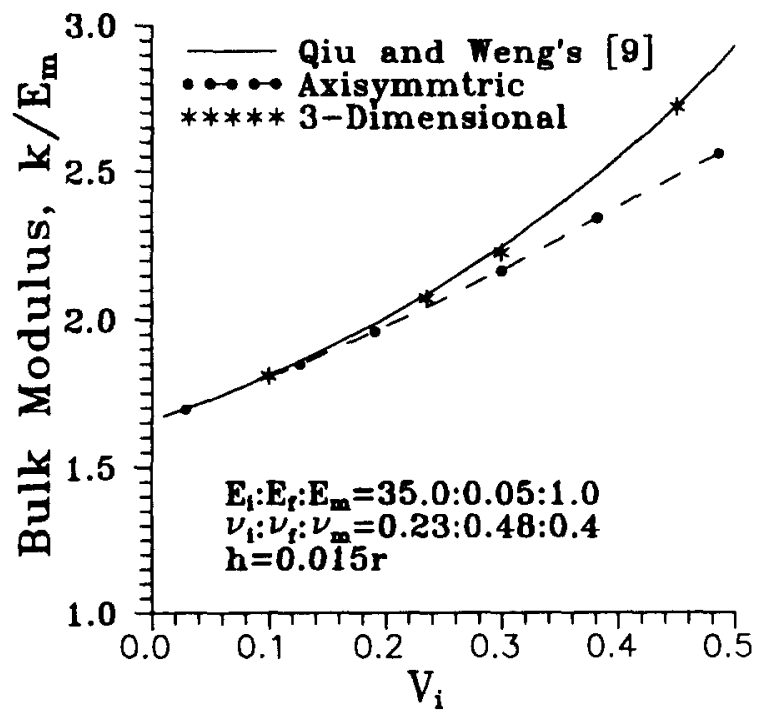

Fig. 12. Comparison of bulk modulus of a three-phase composite vs filler volume fraction predicted by three-dimensional and axisymmetric analyses and Qui and Weng's solution for $h=0.015 r$, and $E_{\mathrm{i}}: E_{\mathrm{f}}: E_{\mathrm{m}}=35.0: 0.05: 1.0, v_{\mathrm{i}}: v_{\mathrm{f}}: v_{\mathrm{m}}=0.23: 0.48: 0.4$

are much higher than those obtained by the threedimensional analysis. The calculations show that the difference in moduli ratios is caused by the difference in the average stress $\bar{\sigma}_{z}$ predicted by the two analyses. This happens because the boundary area used to predict the applied stress $\bar{\sigma}_{z}$ in the axisymmetric analysis is smaller than the area in the three-dimensional analysis, and the stress $\bar{\sigma}_{z}$ calculated from the three-dimensional model is lower than that from the axisymmetric model. The higher the volume fraction, the larger is the difference in the stress $\bar{\sigma}_{z}$ in the two analyses. If in the axisymmetric analysis the boundary area is extended into the same area as used in the three-dimensional analysis, and a proper stress $\bar{\sigma}_{z}$ is added in the extended part, we find that the results of the two analyses can gain a satisfactory agreement.

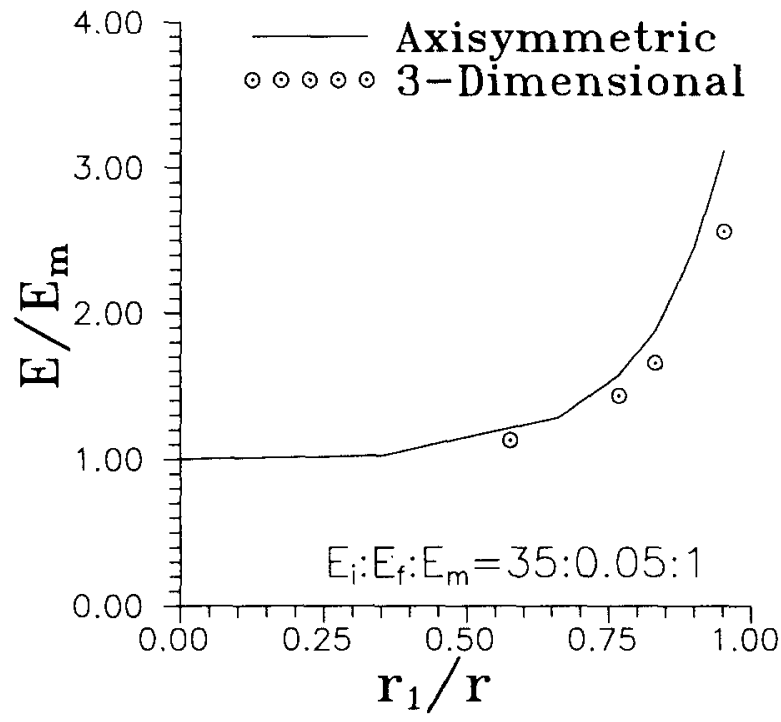

Fig. 13. Comparison of modulus of elasticity of the three-phase composite vs. $r_{1} / r$ predicted by three-dimensional and axisymmetric analyses.

Table 4

Predicted modulus of elasticity of a two-phase composite as a function of $r_{1} / r$ for $E_{\mathrm{i}}=60.4 \times 10^{6} \mathrm{lbf}$ in $^{-2}, E_{\mathrm{m}}=11.8 \times 10^{6} \mathrm{lbf}^{-2}$, $v_{\mathrm{i}}=0.257, v_{\mathrm{m}}=0.197$

\begin{tabular}{lll}
\hline$r_{1} / r$ & $\begin{array}{l}\text { Present modulus of } \\
\text { elasticity }\left(\times 10^{6} \mathrm{lbf} \text { in }{ }^{-2}\right)\end{array}$ & $\begin{array}{l}\text { Agarwal and Broutman's } \\
\text { results }[6]\left(\times 10^{6} \mathrm{lbf} \text { in }\right.\end{array}$
\end{tabular}

\section{Comparison with theoretical results from Refs. $\mid 2,4,6]$}

To investigate the present model more thoroughly, we simply compare our results with the theoretical solutions obtained from Refs. [2,4,6] for two-phase composites. The component properties are taken from Ref. [6]: $E_{\mathrm{i}}=60.4 \times 10^{6} \mathrm{lbf}$ in $^{-2}, v_{\mathrm{i}}=0.257$, and $E_{\mathrm{m}}=$ $11.8 \times 10^{6} \mathrm{lbf}$ in $^{-2}, v_{\mathrm{m}}=0.197$; the interphase property is taken as equal to that of the matrix. Table 4 gives the comparison of the predicted modulus of elasticity between our results and Agarwal and Broutman's results. A small difference in the elastic modulus exists and it may be caused by the difference in dividing forms of the three-dimensional mesh of the cell. Fig. 14 shows the comparisons of the bulk modulus and shear modulus between our results and the theoretical results obtained from Refs. [2,4]. The results show that there is a very good agreement among the three results in bulk modulus, but a great divergence exists in the predicted values of effective shear modulus. Because the determination of shear modulus is related to the approach 

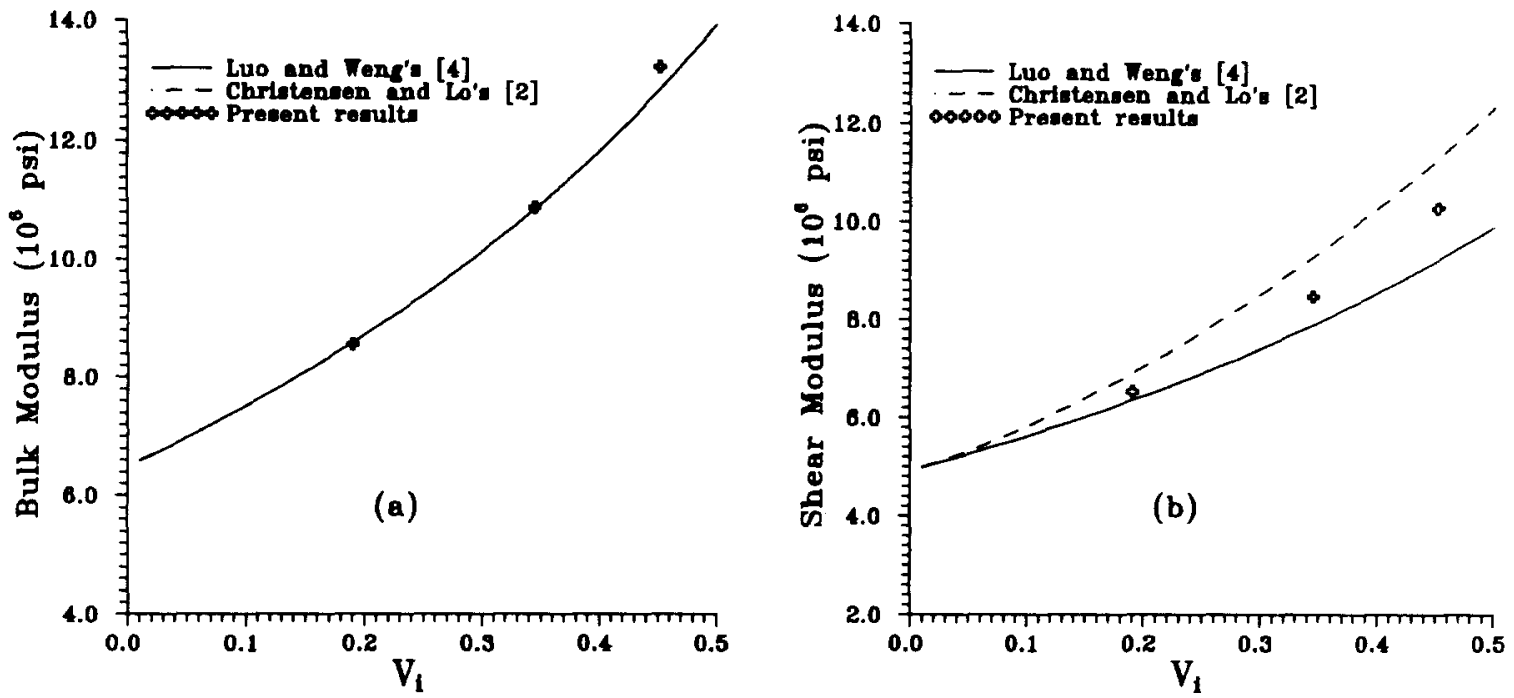

Fig. 14. Comparisons of the predicted (a) bulk and (b) shear moduli of a two-phase composites vs. volume fraction $V_{\mathrm{i}}$ of inclusions obtained in three-dimensional analysis and theoretical solutions for $E_{\mathrm{j}}=60.4 \times 10^{6} \mathrm{lbf} \mathrm{in}^{-2}, E_{\mathrm{m}}=11.8 \times 10^{6} \mathrm{lbf}^{-2}, v_{\mathrm{i}}=0.257, v_{\mathrm{m}}=0.197$.

used, our results which lie between the two theoretical solutions may be considered to be reasonable.

\section{Conclusions}

The results of the three-dimensional analysis and the axisymmetric analysis for stresses around the interface agree very well, even though a few differences exist in some local stress values and stress distributions. The predicted bulk modulus in the three-dimensional analysis agrees well with the theoretical solution for threephase composites but presents a divergence with the predicted modulus in axisymmetric analysis for high volume fractions of inclusions. An investigation of the unit cell structures in the two analyses indicates that this divergence may be attributed to the difference in the unit cell structures between models for high volume fractions of inclusions and can be reduced by properly modifying the structure and stress distribution of the axisymmetric unit cell; the axisymmetric analysis can then give satisfactory results.

The numerical analyses show that the stress distributions around the two interfaces for the three-phase composite are sensitive to the interphase property. The changes in aspect ratios of the interphase and inclusions can also affect the internal stress concentrations and mechanical properties of the material.

A simple comparison of our results with the theoretical solutions $[2,4,6]$ for two-phase composites indicates that the numerical results of the three-dimensional analysis are reasonable. The predicted bulk modulus agrees very well. Because the determination of shear modulus is related to the approach used, the predicted shear modulus lies between the theoretical results, which can be considered to be reasonable.

\section{Acknowledgements}

This work was supported by the National Natural Science Foundation of China and Laboratory for Nonlinear Mechanics of Continuous Media, Institute of Mechanics, Chinese Academy of Sciences.

\section{References}

[1] Z. Hashin, The elastic constants of heterogeneous materials, $J$. Appl. Mech., 29 (1962) 143.

[2] R.M. Christensen and K.H. Lo, Solutions for effective shear properties in three phase sphere and cylinder models, J. Mech. Phys. Solids, 27 (1979) 315.

[3] G.J. Weng, Some elastic properties of reinforced solids, with special reference to isotropic ones containing spherical inclusions, Int. J. Eng. Sci., 22 (1984) 845.

[4] H.A. Luo and G.J. Weng, On Eshelby's inclusion problem in a three-phase spherically concentric solid, and a modification of Mori-Tanaka's method, Mech. Mater., 6 (1987) 347.

[5] T. Mori and K. Tanaka, Average stress in matrix and average elastic energy of materials with misfitting inclusions, Acta Metall., 21 (1973) 571.

[6] B.D. Agarwal and L.J. Broutman, Three-dimensional finite element analysis of spherical particle composites, Fibre Sci. Technol., 7 (1974) 63.

[7] V.A. Matonis and N.C. Small, A macroscopic analysis of composites containing layered spherical inclusions, Polym. Eng. Sci., 9(2) (1969) 90.

[8] J.N. Goodier, Concentration of stress around spherical and cylindrical inclusions and flaws, J. Appl. Mech., 55(7) (1933) 39.

[9] Y.P. Qiu and G.J. Weng, Elastic moduli of thickly coated particle and fiber-reinforced composites, J. Appl. Mech., 58 (1991) 388 .

[10] Z. Hashin and B.W. Rosen, The elastic moduli of fiber-reinforced materials, J. Appl. Mech., 31 (1964) 223.

[11] Y. Benveniste, G.J. Dvorak and T. Chen, Stress fields in composites with coated inclusions, Mech. Mater., 7 (1989) 305.

[12] S. Lu, L. Yan, X. Zhu and Z. Qi, Microdamage and interfacial adhesion in glass bead-filled high density polyethylene, $J$. Mater. Sci., 27 (1992) 4633. 